\title{
INFLUENCE OF PLANT GROWTH PROMOTING RHIZOBACTERIA ON GROWTH AND BIOCHEMICAL PARAMETERS OF CORN (ZEA MAYS L.) VAR. SEEDLINGS
}

\author{
Huma Nawaz \\ Department of Microbiology and Molecular Genetics, University of the Punjab, Quaid-e-Azam Campus, Lahore-54590, Pakistan. \\ Saima Javed \\ Department of Microbiology and Molecular Genetics, University of the Punjab, Quaid-e-Azam Campus, Lahore-54590, Pakistan.
}

\section{Muhammad Faisal*}

Department of Microbiology and Molecular Genetics, University of the Punjab, Quaid-e-Azam Campus, Lahore-54590, Pakistan.

*Corresponding author

Department of Microbiology and Molecular Genetics, University of the Punjab, Quaid-e-Azam Campus, Lahore-54590, Pakistan. Telephone: 924235952811; Email: mohdfaysal@yahoo.com

\begin{abstract}
Impact of inoculations of eight individual strains (CN2, CSH4, Cu35, CMS7, CSH23, CSH27, CF18, and Cu47, two combinations (Comb 4A and Comb 4B) of four Bacillus strains and mixture of all strains (Mix) was studied. Various growth and biochemical parameters of maize plant were determined in pot trials. Strain Bacillus subtilis CN2 showed $49 \%$ maximum increase in shoot length. The blend of entire strains was observed to have their lenghth increased about $21 \%$. Bacillus subtilis CN2 showed maximum increase 155 $\%$, in fresh biomass and $233 \%$ increase in dry weight. Bacillus amyloliquefaciens (Cu47) and Comb 4A and showed maximum peroxidase content of $163 \%$ and $94 \%$ as compared to uninoculated control. Comb $4 \mathrm{~B}$ was shown to have significant higher content of acid phosphatase $(811 \%)$. Increase in all other physical and biochemical parameters were noticed. Therefore, Bacillus strains exhibited characteristic increased potential of plant growth and can have great application in innovative agricultural practices.
\end{abstract}

Keywords: Bacteria, corn, auxin, Bacillus, acid phosphatase, peroxidase

\section{Introduction}

Plants are valuable natural assets and mankind's existence depends on these natural resources. Plants and microbes are in the everlasting arms race to retain their predominance within their distinct niche. These interactions have agronomic importance because of rhizoremediation, enhance crop yield and eradication of phyto-pathogens. Globally, maize is most significant and economically important after rice and wheat. Maize 
yield has been having decreasing due to depletion of nutrients led to poor land fertility (Adjanohoun et al., 2011). Plant development and improvement implicates a compact synchronization of structure with the successive association of cell division, extension and separation. These occasions require chemical communication between the shoot and root, which can be influenced by both biotic and abiotic elements. Plants devote a significant quantity of their carbon sources (dead cells, sugars, proteins) to rhizosphere microbiota to get microbial benefits for improving plant development (Pieterse et al., 2016). Soil-plantmicro-organism collaboration allows the exchange of signals, can allow plants to overcome such challenges, and prompts the advancement of innovative agricultural uses (Ortíz-Castro et al., 2009). Plants genotypic traits and quorum sensing involved in microbiome formation. Microbes in the rhizosphere enhance mineral and water uptake, providing growth regulators. Several groups of plant- associated microbes include rhizobacteria which have the ability to enhance plant growth (PGPR: Plant growth-promoting) and stimulate plant defenses.

PGPR species include Azospirillum, Bacillus, Pseudomonas and Enterobacteria. Bacillus sp. activate pathways undergo cytological alternation to protect and promote plant growth (Kloepper et al., 2004). Several Bacillus strains belonging to the different groups provide salt stress tolerance, insect protects and act as biocontrol agents. Bacillus species produces metabolites to antagonize Phyto-pathogens. Some species individually act as stimulators and bio controllers. Many species produce effective results in combination with other species. Microbiomes will contribute to plan the microbial agriculture, to enhance crop production with lesser use of harmful pesticides (Pieterse et al., 2016). The purpose of our research was to check the influence of plant-microbe interaction on growth promotion of plant including various Bacillus species in maize plants.

\section{Materials and Methods}

\section{Bacterial Strains}

Eight bacterial strains of Bacillus species were used in the current study which were previously isolated and identified as Bacillus subtilis CN2, Bacillus safensis Cu35, Bacillus pumilus CSH4, Bacillus sonorensis CSH27, Bacillus cereus CMS7, Bacillus sp. CSH23, Bacillus thuringiensis Cu47, Bacillus axarquiensis $\mathrm{CF} 18$. These strains were routinely maintained on L-agar.

\section{Plant-Microbe interaction}

Certified maize kernels (var. Neelum) were collected from seed Corporation, Punjab Lahore.

\section{Seed Sterization and Inoculation}

The surface sterization of corn seeds was carried out for 5 minutes with $0.1 \% \mathrm{HgCl}_{2}$ solution. The kernels were washed with distilled water five times. For inoculation, seeds were suspended and stirred in 
each bacterial suspension cultures (mention above) separately, with combinations of Comb 4A (CSH4, CSH27, Cu47 and CF18) and Comb 4B (CSH23, Cu35, CN2 and CMS7) and a blend of entire strains for 30 minutes. Optical density was adjusted to 1 at $600 \mathrm{~nm}$ to confirm the same amount of cells for inoculation. Seeds with no inoculation were used as control.

\section{Pot experiment}

The wire house was used for pot trials in the Department of MMG, PU, Lahore to check the effect of bacteria on Maize plant. These trials were designed to observe the response of direct inoculations of eight individual and two combination (4A and $4 \mathrm{~b}$ ) mixtures of bacterial strains on corn seeds under natural environmental conditions. Natural garden-sieved soil from field area was collected and filled in each pot. Every plant that was inoculated and those which were placed in control group were arranged in triplicate. Ten inoculated seeds were sowed and pots were arranged in randomized pattern. After six weeks of sowing of seeds, thinning around six plants was done. After maturation, harvestion was done. Various physical growth parameters and biochemical parameters were noted. Un-inoculated control experiment was also run in parallel.

\section{Measurement of growth parameters}

Duration of seed sowing was six months from February 2015 to July 2015. Various parameters like length of shoot and cob, Weight of cob, biomass of fresh and dry plant were measured and results were observed.

\section{Measurement of biochemical parameters}

Different biochemical analysis were performed to determine the amount of phenolic compounds, peroxidase content, acid phosphatase activity, malionaldehyde (MDA) in leaves and pigment analysis.

\section{Total phenolic content}

Total phenolic content was evaluated by using $1 \mathrm{ml}$ of aqueous extract of plant and dissolving in 5 $\mathrm{ml}$ of Folin ciocaleu reagent and then with $4 \mathrm{ml}$ of $1 \mathrm{M}$ sodium carbonate. After one hour, absorption was measured in the $765 \mathrm{~nm}$. Total amount of phenolic compounds was accounted by using following formula: $\mathrm{C}=\mathrm{c} . \mathrm{V} / \mathrm{m}$ in Gallic acid equivalents (GAE) (Agbor et al., 2014).

\section{Peroxidase activity}

Crushed and frozen plant biomass was used to determine peroxidase activity. Plant material was added in $4 \mathrm{ml}$ phosphate buffer saline after which centrifugation at $14,000 \mathrm{rpm}$ for 10 minutes at $4^{\circ} \mathrm{C}$ was done. Supernatant was stored for enzyme assay. Quantitative measurement of peroxidase was done by using (David and Murray 1965) method. 


\section{Acid phosphatase content}

Acid phosphatase content was determined by using the frozen plant material. Activity of corn leaf samples was determined by using the method of (Iqbal and Rafique, 1987) for enzyme quantification and extraction.

\section{Malionaldehyde content estimation}

Thiobarbituric acid (TBA) method was used for the estimation of malionaldehyde. $0.5 \mathrm{~g}$ of icecovered leaves were dissolved in $5 \mathrm{ml}$ of $5 \%$ trichloroacetic acid (TCA) with centrifugation at 10,000 rpm for 10 minutes. $3 \mathrm{ml}$ of malionaldehyde extraction solution in supernantent was mixed robustly with $3 \mathrm{ml}$ of thiobarbituric acid solution $(0.5 \%)$. The mixture obtained was then boiled at $95^{\circ} \mathrm{C}$ for half hour in water bath and then cooled at room temperature. The mixture was then centrifuged for 15 minutes at 10,000 rpm. Absorbance was measured at 450, 532 and $600 \mathrm{~nm}$ with spectrophotometer. Malionaldehyde concentration was estimated by applying the formula MDA $(\mu \mathrm{mol} / \mathrm{ml})=6.45 \times\left(\mathrm{D}_{532}-\mathrm{D}_{600}\right)-0.56 \times \mathrm{D}_{450}$.

\section{Estimation of photosynthetic pigments}

Frozen crushed leaf samples which were homogenized using $80 \%$ acetone were used to measure photosynthetic pigments like chlorophyll-a, chlorophyll-b and carotenoid content on different absorbance values according to Arnon et al (1949).

\section{Results}

\section{Plant growth Parameters}

\section{Shoot length}

Bacterial inoculations improved the growth of maize plant. Bacillus subtilis CN2 showed 49\% increase in shoot length. In the same way, the strain mixture was observed to increase the length of about 21 $\%$. Strains CSH4, Cu35, Comb 4A and Comb 4B resulted in $32 \%, 27 \%, 9 \%$ and $8 \%$ gain in elevation respectively whereas $\mathrm{CSH} 27, \mathrm{Cu} 47, \mathrm{CF} 18$ and $\mathrm{CSH} 23$ resulted in reduction of shoot length in contrast to control plants. CMS7 exhibited no change in shoot length in comparison to control plants (Figure 1).

\section{Length of cob}

All plants showed significant increase in length except CSH23 inoculated plant. There was a slight increase in cob length with CSH4 inoculation. Surprizingly, Bacillus sonorensis strain CSH27 showed 150 $\%$ increase in the length of cob as compared with control. Furthermore, plants inoculated with strains $\mathrm{Cu} 47$, $\mathrm{CF} 18, \mathrm{Cu} 35, \mathrm{CN} 2, \mathrm{CMS} 7, \mathrm{Comb} 4 \mathrm{~A}, \mathrm{Comb} 4 \mathrm{~B}$ and the entire strain mixture showed $53 \%, 112 \%, 20 \%$, $126 \%, 93 \%, 75 \%, 94 \%$ and $63 \%$ rise in length of the cob respectively as oppose to control (Figure 2). 
120

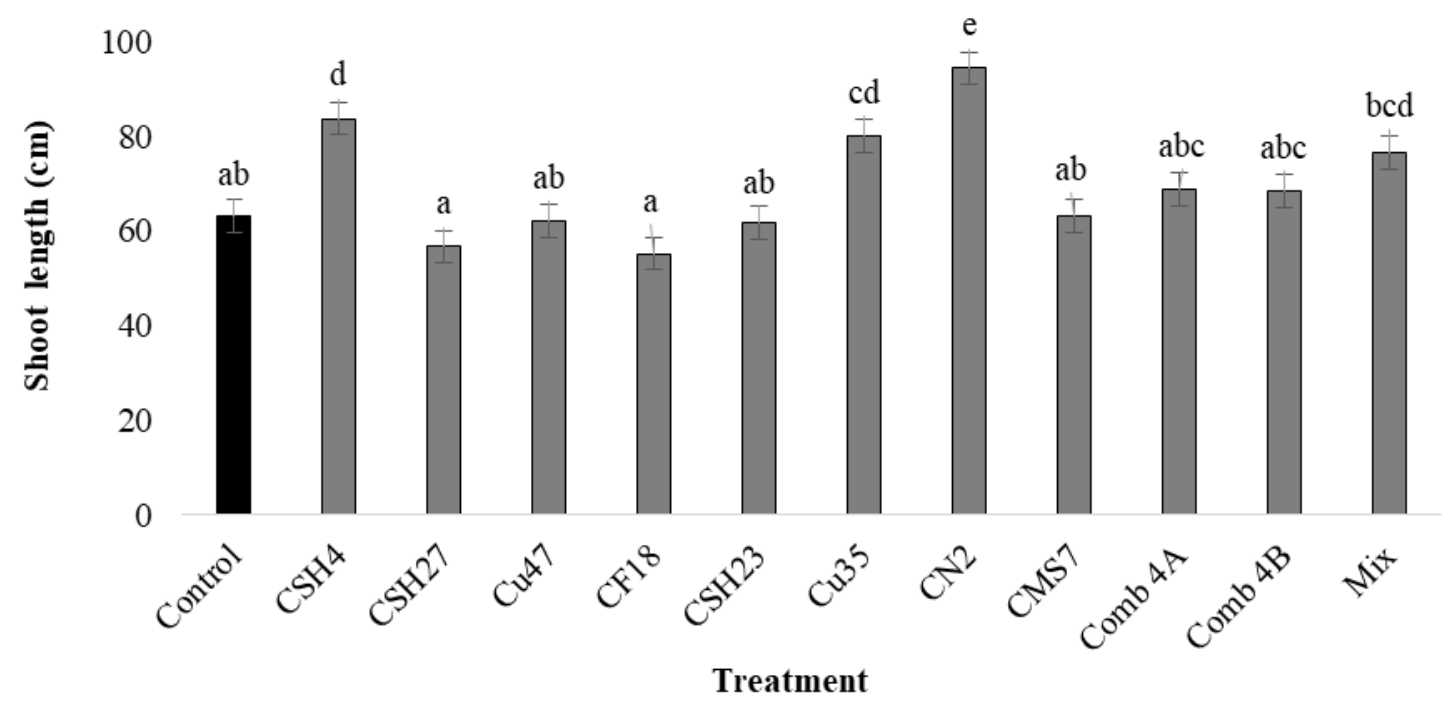

Figure 1: Effect of bacterial strains on shoot length of maize (pot experiment, $n=3 \pm S E M$ of the mean).

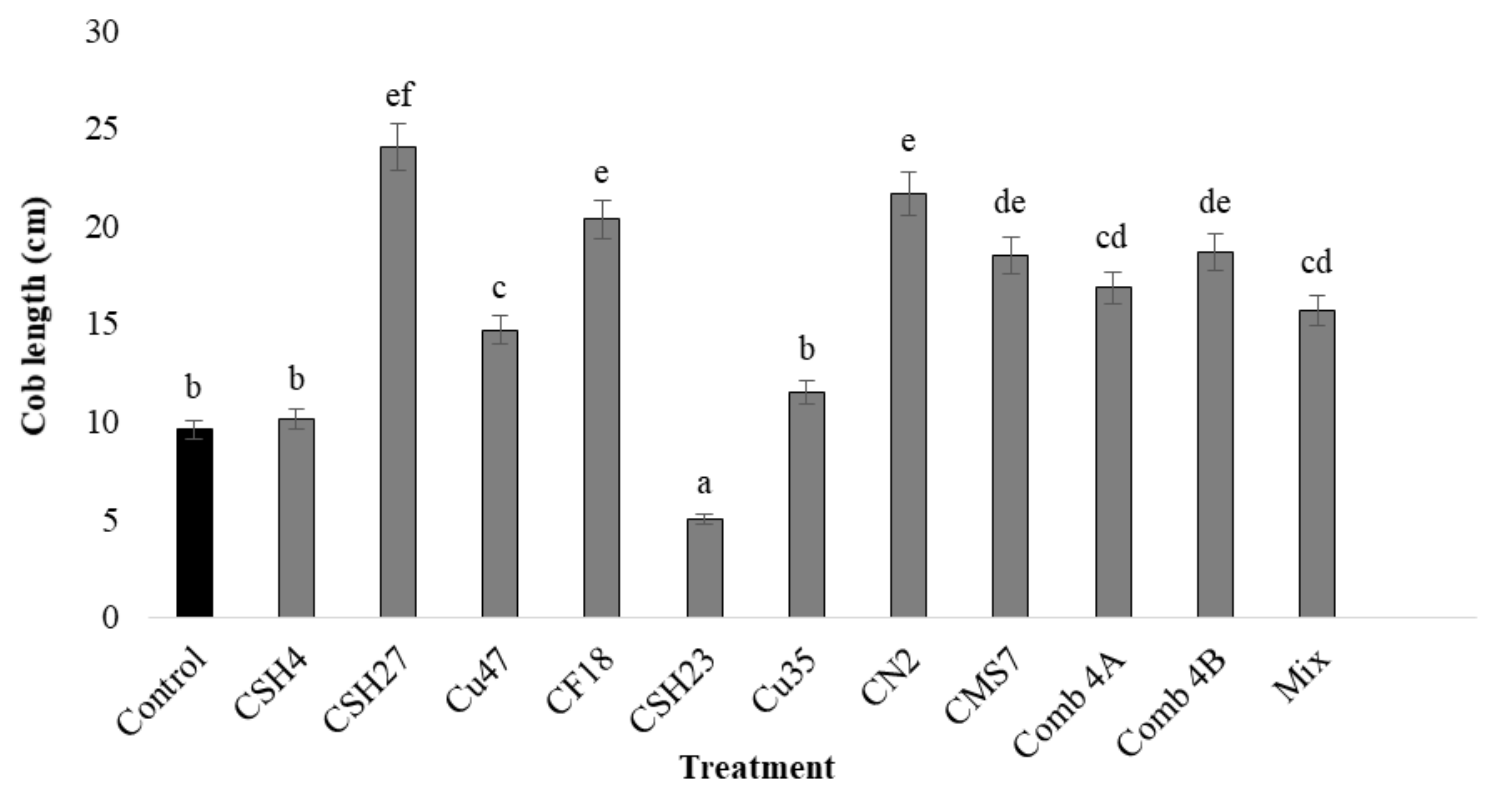

Figure 2: Effect of bacterial strains on cob length in maize (pot experiment, $n=3 \pm S E M$ of the mean). 


\section{Weight of Cob}

A significant gain in weight of cob was observed after bacterial inoculation. Bacillus sonorensis CSH27 showed (285 \%) and CF18 (207\%) increase as compared to control plants. Spike weight also increased significantly with $115 \%, 131 \%, 115 \%$ and $85 \%$ when strains CN2, Comb 4A, Comb 4B and Strain mixture were applied respectively. Conversely, strains CSH4, Cu47, CSH23 and CMS7 significantly reduced the weights of the spikes compared to control (Figure 3).

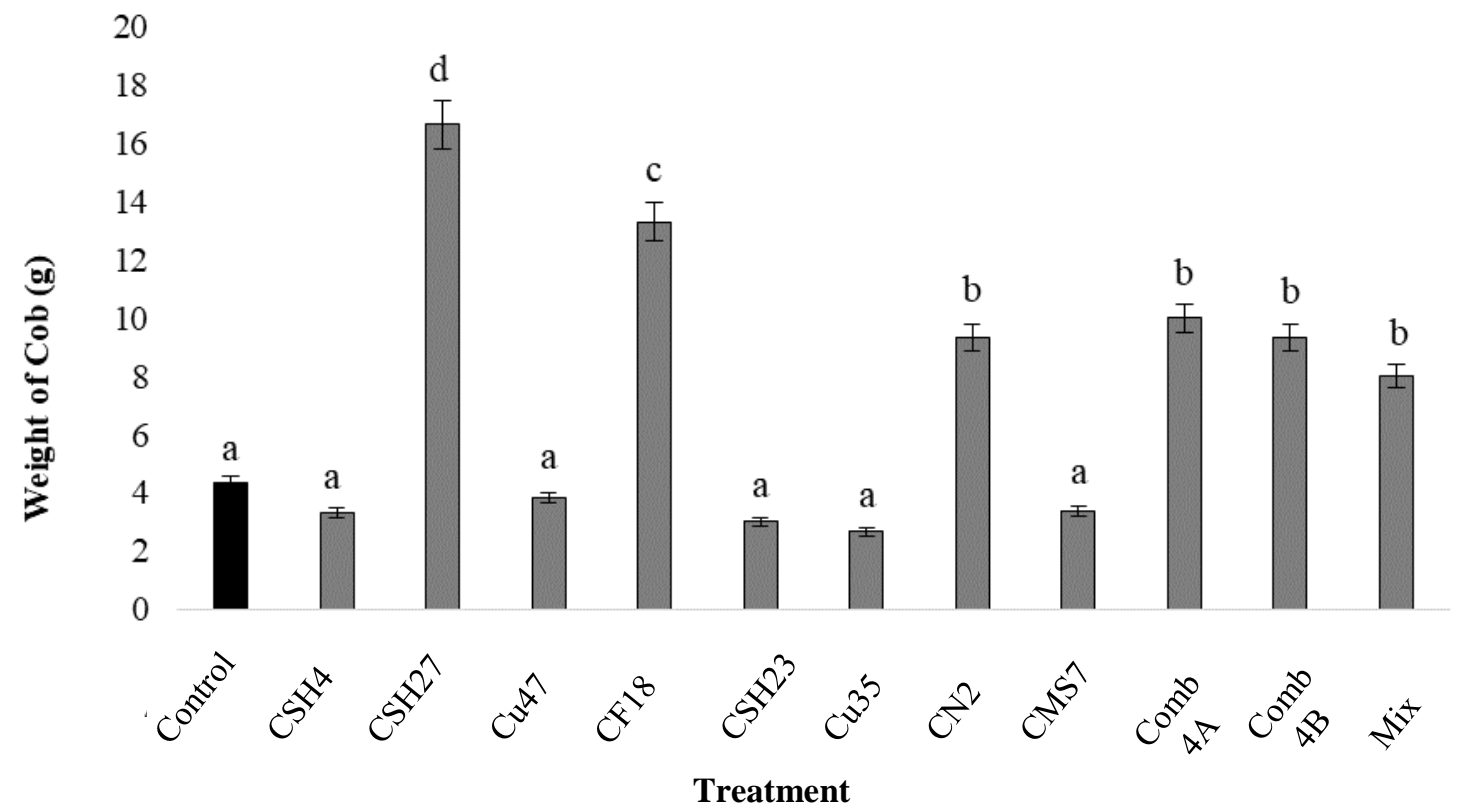

Figure 3: Effect of bacterial strains on cob weight in maize (pot experiment, $n=3 \pm S E M$ of the mean).

\section{Fresh and Dry Biomass}

All the Maize plants that were inoculated were observed to have their fresh weight improved and increased except with treatment with strain CMS7. All other inoculated plants by strains CSH4, CSH27, $\mathrm{Cu} 47, \mathrm{CF} 18, \mathrm{CSH} 23, \mathrm{Cu} 35, \mathrm{CN} 2, \mathrm{Comb} 4 \mathrm{~A}, \mathrm{Comb} 4 \mathrm{~B}$ and strain mix showed 7\%, $73 \%, 125 \%, 87 \%, 27$ $\%, 67 \%, 155 \%, 45 \%, 72 \%$ and $36 \%$ respectively induced a significant increase in the fresh corn plant biomass as compared with control plants.

All strains showed increase in dry weight except strains CMS7 and CSH4 inoculated plants. A significant gain in plant dry weight was observed with strains CSH27, Cu47, CF18, CSH23, Cu35, CN2, Comb 4A, Comb 4B and strain mixture with $40 \%, 167 \%, 93 \%, 27 \%, 53 \%, 233 \%, 43 \%, 97 \%$ and $33 \%$ 
increase respectively as compared with control plants. Remarkable gain of $233 \%$ in plant dry biomass was shown by strain CN2 inoculated plants (Figure 4).

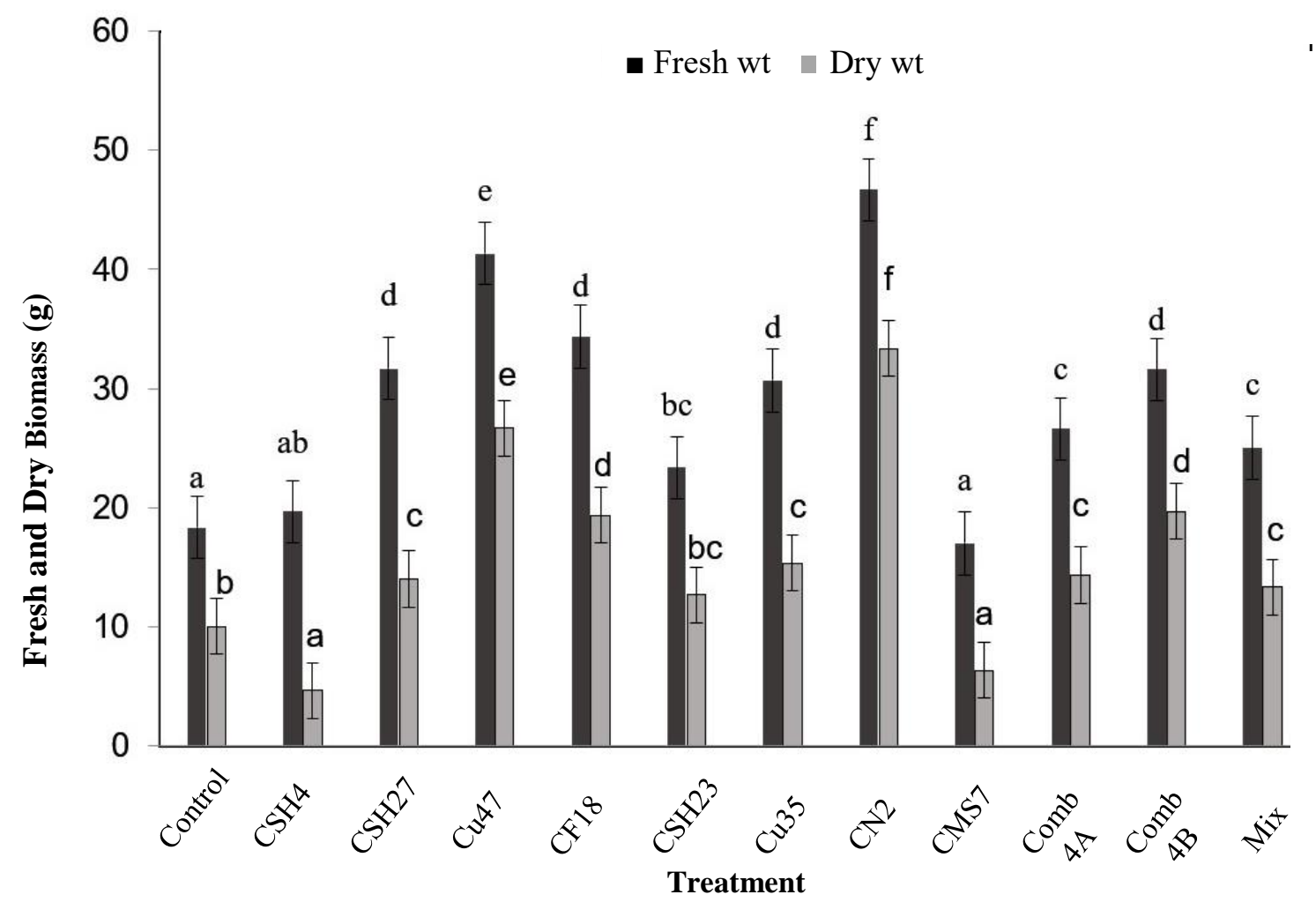

Figure 4: Effect of bacterial strains on fresh and dry weight of maize plant (pot experiment, $n=3 \pm$ SEM of the mean). Different letters on bars are showing significant difference in means, ANOVA followed by Duncan at $95 \%$ confidence level.

\section{Biochemical Characterization}

\section{Phenolic compounds}

Bacterial strains were observed to greatly affect the biochemical parameters in maize plant. The strains CSH4, Cu47, CF18, CN2 and CMS7 (16.91 mg/g) showed no increase in phenolic content while strains CSH27, CSH23, Cu35 (16.92 mg/ ml), Comb 4A, Comb 4B and strain mixture (16.93, 16.94, 16.94 $\mathrm{mg} / \mathrm{g}$ ) showed slightly increase in amount of phenols as compared to control (Figure 5). 


\section{Peroxidase content}

Considerably increased amounts of peroxidase activity of $163 \%$ was observed in all the plants that were inoculated with strain $\mathrm{Cu} 47$ followed by strain CSH23 and Comb 4A with $107 \%$ and $94 \%$ increase in peroxidase activity respectively. A significant increase of $25 \%, 74 \%, 55 \%, 46 \%, 49 \%, 52 \%$ and $55 \%$ was also observed with other strains CSH27, CF18, Cu35, CN2, CMS7, Comb 4B and strain mixture respectively. Plants inoculated with strain CSH4 showed activity slightly less than the control. Strains Comb 4A showed more increase than Comb 4B and strain mixture when compared to control (Figure 6).

\section{Acid phosphatase}

Comb 4B was shown to have significant higher amounts of acid phosphatase compared to plant control. Strains Cu47, CF18, CSH23, CN2, CMS7 and Comb 4A inoculated plants showed stimulatory effect of $55 \%, 244 \%, 147 \%, 89 \%, 27 \%$ and $131 \%$ respectively on enzyme content. CSH4, CSH27, Cu35 and Mix inoculated plants revealed no rise in acid phosphatase content in contrast to un-inoculated control plants (Figure 7).

\section{Malionaldehyde content in leaves}

The level of Malionaldehyde (MDA) in leaves was evaluated of inoculated and control plants. In all inoculated plants $\mathrm{Cu} 35$ presented rise in malionaldehyde content of $291 \%$ than the un-inoculated control plants. Strains CSH4, CSH27, Cu47, CF18, CSH23, CN2, CMS7, Comb 4A, Comb 4B and strain mixture inoculated plants were shown to have decreased content of MDA relative to the pants placed in control group (Figure 8). 


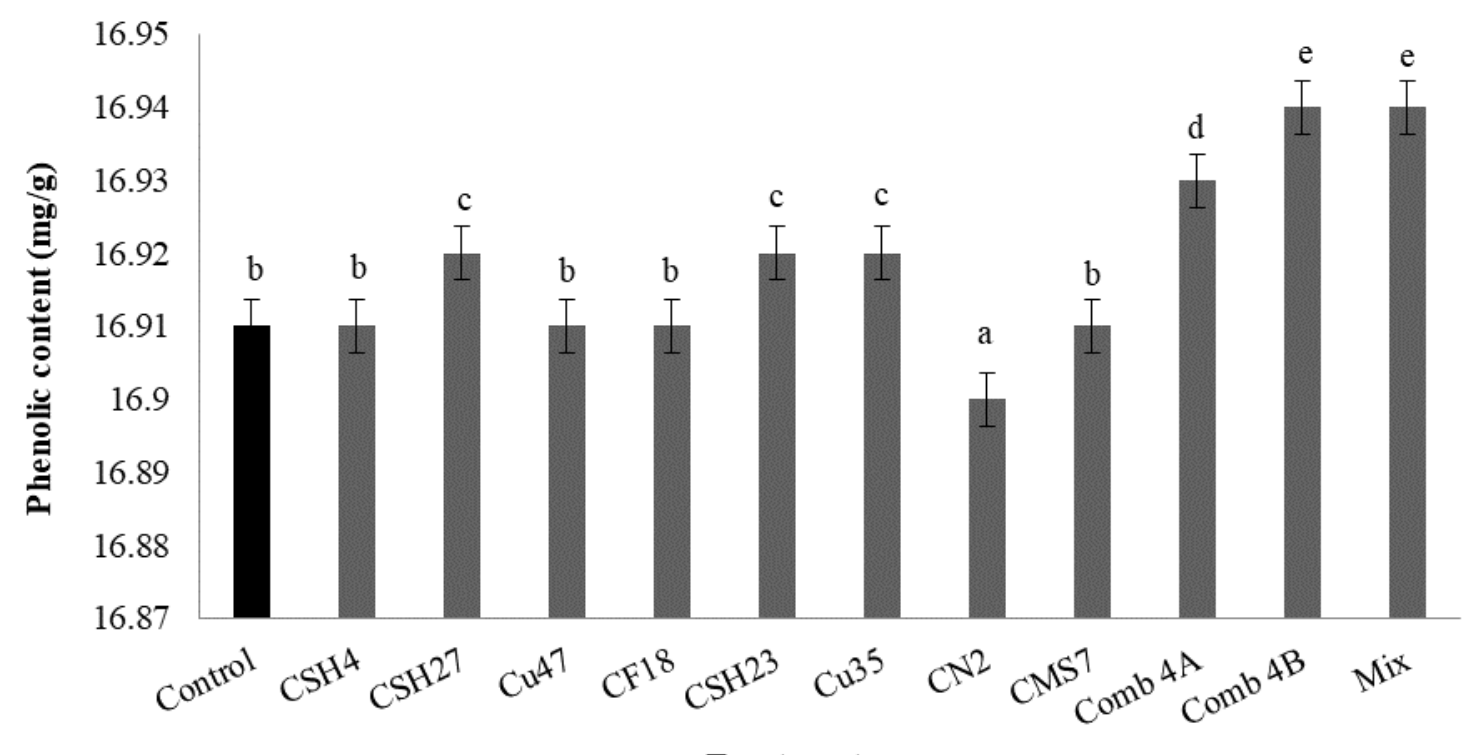

Treatment

Figure 5: Effect of bacterial strains on phenolic content of corn plants (pot experiment, $n=3 \pm$ SEM of the mean).

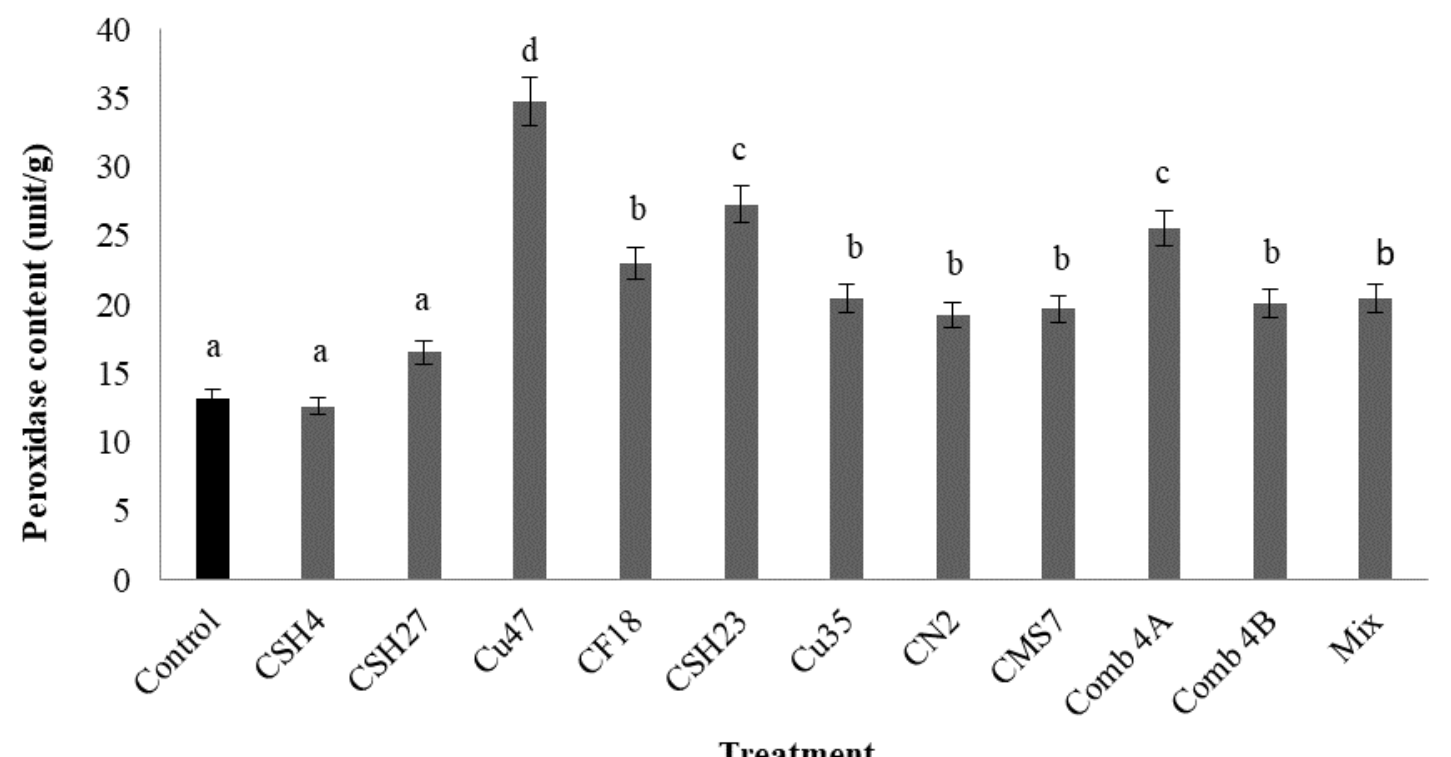

Figure 6: Effect of bacterial strains on peroxidase activity of corn leaves (pot experiment, $n=3 \pm$ SEM of the mean). 


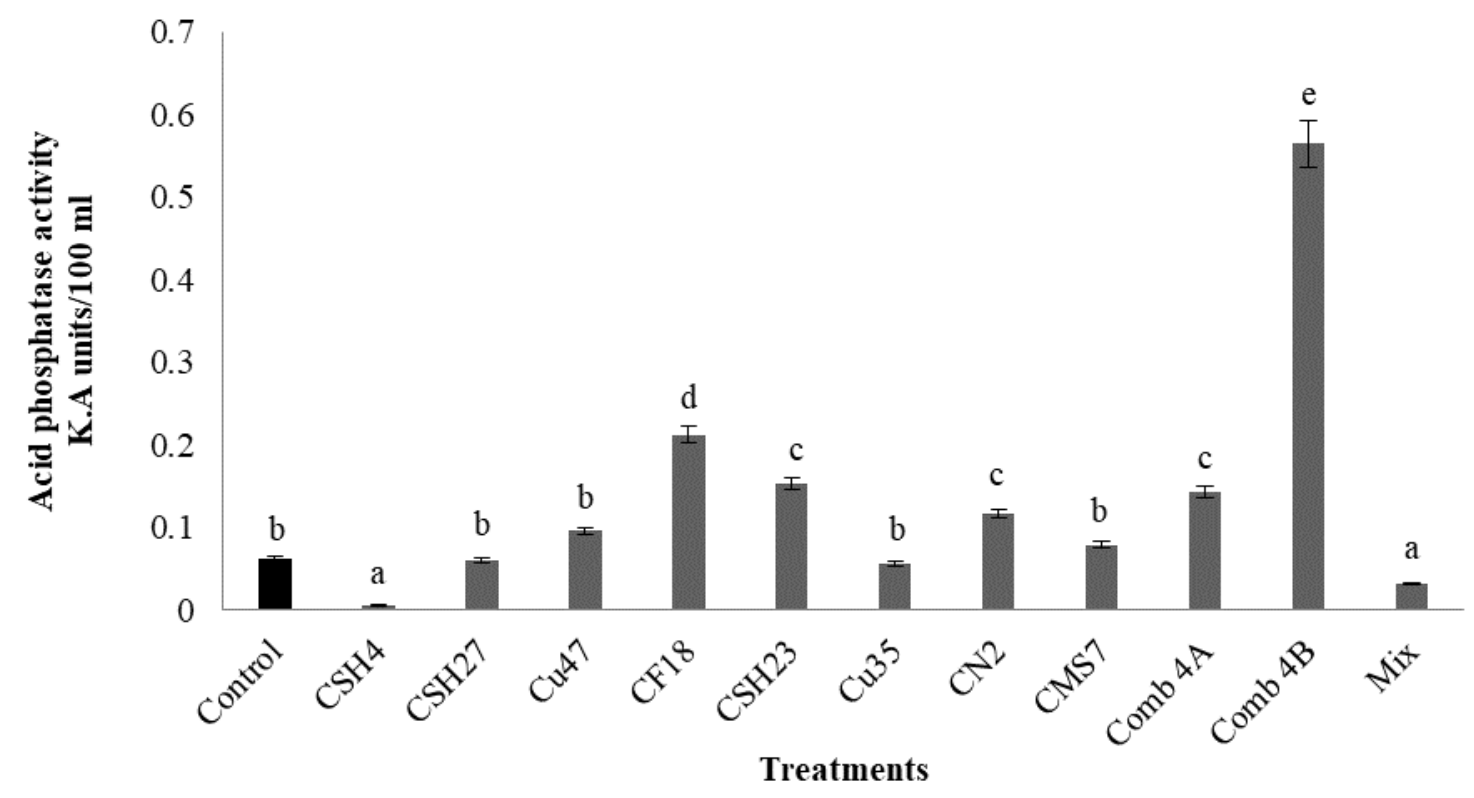

Figure 7: Effect of bacterial strains on acid phosphatase content of wheat leaves (pot experiment, $n=3 \pm$ SEM of the mean).

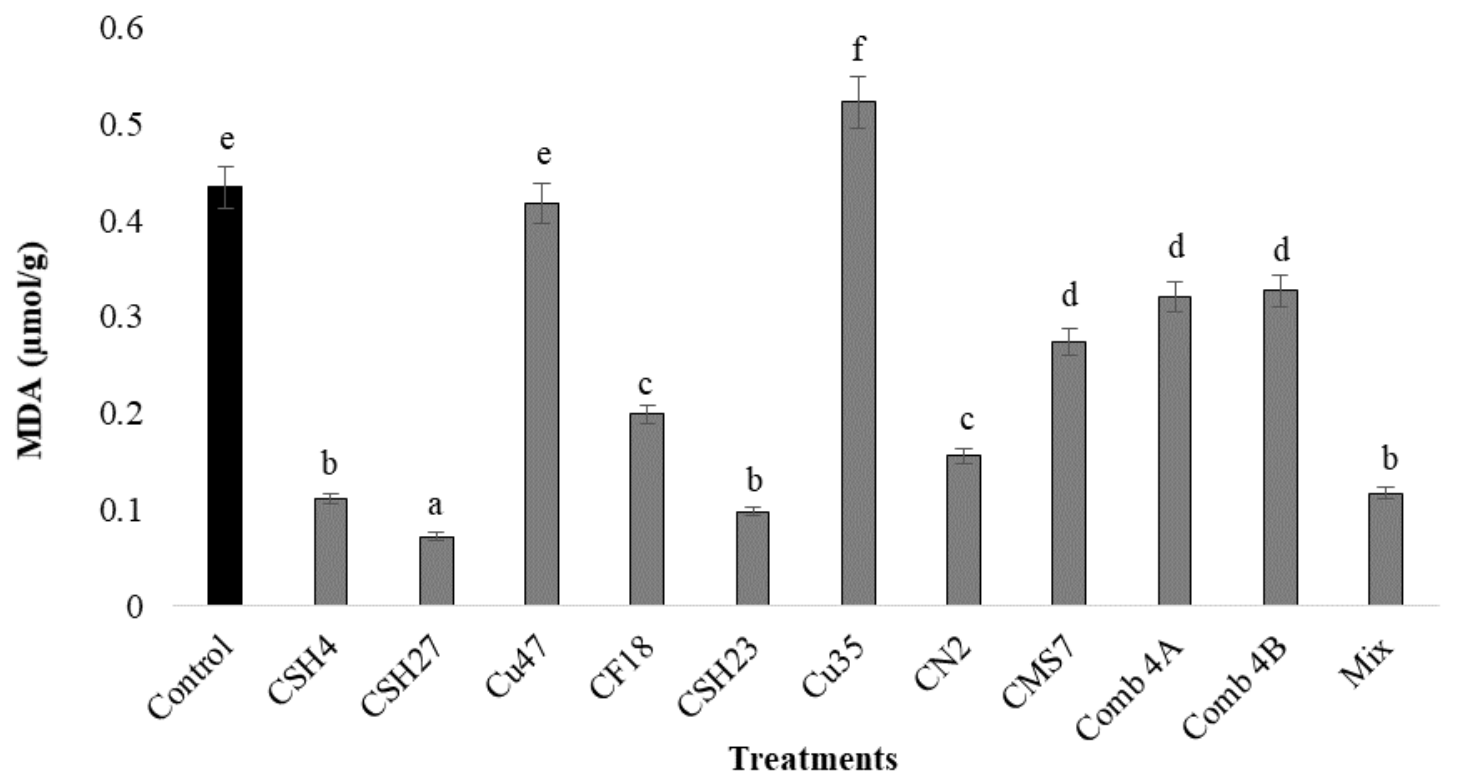

Figure 8: Effect of bacterial strains on MDA contents of maize plants (pot experiment, $n=3 \pm$ SEM of the mean). 


\section{Pigment content in maize plant}

Inoculated plants with strains CSH4, CSH27, Cu47 and CMS7 exhibited 44 \%, 35\%, $38 \%$ and 35 $\%$ increase in chlorophyll ' $\mathrm{a}$ ' content while strains $\mathrm{CF} 18, \mathrm{Cu} 35, \mathrm{CN} 2$, Comb 4A, Comb 4B and strain mixture represented reduction in chlorophyll-a content in comparison to control plants. Chlorophyll-b was observed to be higher in strains $\mathrm{CSH} 27, \mathrm{CF} 18$, Comb 4A, Comb 4B and strain mixture with $22 \%, 27 \%, 14 \%, 14 \%$, $26 \%$ and $23 \%$ increase respectively. Conversely, the other inoculated plants exhibited decreased content of 'chlorophyll-b' relatively to control plants. Carotenoid amount was greater in leaf extracts of inoculated plants. Cu35 showed remarkable increase (51\%) than control plants. Cu47, CF18, CSH23, CN2, Comb 4A, Comb 4B and Mix inoculated plants showed $17 \%, 13 \%, 5 \%, 27 \%, 17 \%, 34 \%$ and $22 \%$ increase while CSH4, CSH27 and CMS7 displayed reduced levels of carotenoid content in comparison to un-inoculated control plants (Figure 9).

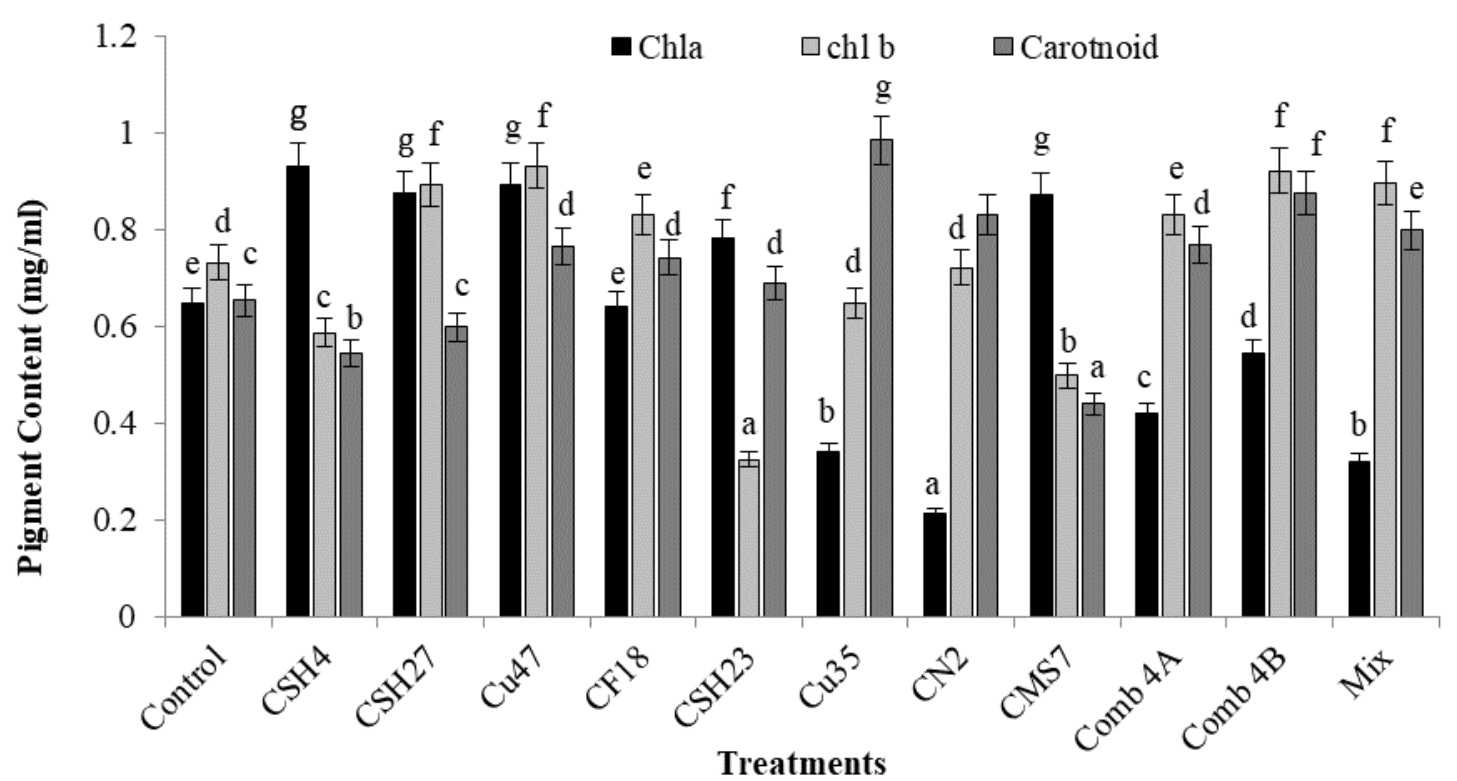

Figure 9: Effect of bacterial strains on chlorophyll a, b and carotenoid content in maize plants (pot experiment, $n=3 \pm S E M$ of the mean).

\section{Discussion}

In our present study, different Rhizospheric Bacillus strains significantly increased development of corn seedlings in contrast to control plants. The above mentioned palnts were examined to have siginifacnt 
results in the enhancement of growth of plants and a significantly activity had been exhibited by most of the strains in various biochemical parameters of corn plants. The strains either used in combination or individually showed coherent activity because these bacteria work in colloboartion. In some parameters (number of cobs, acid phosphatase, MDA) plants inoculated with all strains showed less activity than individual and combination of four strains because of microbial competition.

Cultivation depends on plant-microbe interaction that is advantageous for potential productivity. It was observed in laboratory and field studies that Bacillus and Pseudomonas are useful in plant growth and can be stored for a long time with suitable formulations (Nkebiwe et al., 2016). In rhizosphere PGPR bacteria accomplished plant progression through various channels that comprise enhancement of plant nutrition, production and regulation of phyto-hormones, and elimination of plant pathogens (Martinez-Viveros et al., 2010). Maize is a vital staple harvest in the world and is vulnerable to diseases (Collins and Duffy, 2016).

All the inoculated plants exhibited rise in plant shoot length in comparison to control. Plants with strain mixture showed a $21 \%$ increase in shoot length except Bacillus sonorensis, Bacillus thuringiensis, Bacillus axarquiensis and Bacillus sp. Strains CSH4, Cu35, Comb 4A, and Comb 4B resulted in $32 \%, 27$ $\%, 9 \%$ and $8 \%$ increase in height respectively. Bacillus subtilis showed remarkable increase of $48.8 \%$. Previous studies showed that PGPR bacteria are involved in increased shoot length (Gholami et al., 2009) and PGPR Bacillus strains significantly promote growth of the plant (Kuan et al., 2016). Most of the strains showed significant increase in cob length. Bacillus sonorensis showed remarkable increased $150 \%$, Comb 4A (75\%), Comb 4B (94\%) and strain mixture $(63 \%)$, respectively. Our result is supported by similar finding that there is significant increase in the cob length and yield of corn by bacteria as $58.3 \%$ and $27.4 \%$ (Shanmugam et al., 2011). In recent studies, various combinations of microbial strains (Funneliformis mosseae and Bacillus sonorensis) showed strong growth promoting activity by decreasing the use of chemical fertilizers (Thilagar et al., 2016). In the current study, all the strains showed increase in fresh weight as Bacillus subtilis showed $46.66 \%$ increase, combinations also showed $26 \%, 36 \%$ increase as compared to control. Several studies showed Bacillus subtilis and Bacillus amyloliqueficians acts as growth stimulators (Liu et al., 2016).

All strains expect two showed increase in dry weight as Bacillus thuringiensis Cu47, Bacillus subtilis CN2 showed a maximum increase, combinations also showed increase as compared to control.the antioxidant properties of plants can be partialy attributed to the the presence of phenolic compounds (Shalaby and Horwitz, 2015).

Combinations and mixture of strains showed comparatively increased amounts of phenolic contents of $16.94 \mathrm{mg} / \mathrm{g}$ whereas no significant increased has been observed in other strains. However, some studies have indicated that Bacillus species do induced antioxidants properties (Ndeddy Aka and Babalola, 2016). 
There is a remarkable increase in peroxidase activity as Bacillus thuringiensis (Cu47) showed about $163 \%$ and Bacillus sp. showed $107 \%$ increase, Comb 4A, 4B, and strain mixture also indicated major increase in peroxidase activity as compared to control. In recent studies, Bacillus thuringienesis improve drought tolerance, increase nutrient uptake and phosphate solubilization (Armada et al., 2015). It is reported that in plants peroxidases can play a role of catalysts in the production of oxygen reactive species. These are also responsible for the oxidation of some compunds involved in the defense system of plants such as indole acetic acid and other related compounds. These enzymes are the active players in plants growth (Camejo et al., 2016). Combinations (Comb 4B) showed a remarkable increase in enzyme content (811\%). Bacillus axarquiensis $\mathrm{Cu} 47$ showed increase of about $243 \%$ in acid phosphatase content. Previous studies indicated the role of Bacillus axarquiensis in plant phosphate solubilization (Saha et al., 2016). It was shown that phosphate solubilization increased with increase in acid phosphatase activity of bacterial inoculated plants (Tian and Liao, 2015). Bacillus safensi showed an increase of $291 \%$ in MDA content. Conversely, combinations (4A and 4B) and strain mixture inoculated plants showed decrease malionaldehyde content in corn leaves. Malionaldehyde is formed by autooxidation and degradation of fatty acids in cells. The estimation of malionaldehyde in leaves helps to measure lipid peroxidation (Ye et al., 2016).

Chlorophyll content is a direct related with plant growth. Some inoculated plants showed an increase in amount of photosynthetic pigments (chlorophyll-a, chlorophyll-b and carotenoids). It has been reported that growth promoting bacteria can be used to maximize the chlorophyll content of plants up to $50 \%$ (Bal et al., 2012). In this study, Bacillus pumilus showed $44 \%$ increase in chlorophyll-a content. Furthermore, in another study, it was shown that Bacillus subtilis could increase plant photosynthetic activities by increasing leaf photosynthetic proficiency and chlorophyll content (Li et al., 2016). Bacillus thuringienesis and Comb 4B showed maximum increase in chlorophyll-b content. In Comb 4A, Comb 4B and strain mixture showed 14\%, 26\% and 23\% increase. Recent studies indicate that Bacillus thuringienesis is involved in increasing chlorophyll content (Babu et al., 2013). Carotenoid content was found to be higher in leaf extracts of all the inoculated plants. In the present study, strain Cu35 showed remarkable increase of $51 \%$. Plant growth stimulating microorganisms increase pigment content in several plants (Vafadar et al., 2014).

\section{Conclusion}

Hence, from results it is evident that inoculations with individual and combination of four bacterial strains increase the growth and biochemical parameters of plants because of symbiotic relationship between different strains. Plants inoculated with all eight strains (mix) showed less increase in growth parameters than combinational strains because of microbial competition. Microbes are stronger competitors (Vries and Bardgett, 2016). Previous studies showed that microbial communities determine plant-soil feedback (Ke et al., 2015). These bacterial strains can be utilized for promoting the growth of other field crops in order to 
tolerate ecological stress and enhance yield production. In such a way, agricultural countries like Pakistan may get benefit from potential indigenous bacterial strains for increasing soil fertility and better crop cultivation.

\section{Acknowledgement}

This research was part of the $\mathrm{PhD}$ thesis of Dr Huma Nawaz.

\section{References}

Adjanohoun, A., Allagbe, M., Noumavo, P., Gotoechan-Hodonou, H., Sikirou, R., Dossa, K., Glelekakaï, R., Kotchoni, S. \& Baba-Moussa, L. 2011. Effects of plant growth promoting rhizobacteria on field grown maize. Journal of Animal \& Plant Sciences, 11, 1457-1465.

Armada, E., Probanza, A., Roldán, A. \& Azcón, R. 2015. Native plant growth promoting bacteria Bacillus thuringiensis and mixed or individual mycorrhizal species improved drought tolerance and oxidative metabolism in Lavandula dentata plants. Journal of Plant Physiology, 192,1-12.

Arnon, D. I. 1949. Copper enzymes in isolated chloroplasts. Polyphenoloxidase in Beta vulgaris. Plant Physiology, 24, 1.

Babu, A. G., Kim, J.-D. \& Oh, B.-T. 2013. Enhancement of heavy metal phytoremediation by Alnus firma with endophytic Bacillus thuringiensis GDB-1. Journal of Hazardous Materials, 250, 477-483.

Camejo, D., Guzmán-Cedeño, Á. \& Moreno, A. 2016. Reactive oxygen species, essential molecules, during plant-pathogen interactions. Plant Physiology and Biochemistry, 103, 10-23.

Collins, O. C. \& Duffy, K. J. 2016. Optimal control of maize foliar diseases using the plants population dynamics. Acta Agriculturae Scandinavica, Section B-Soil \& Plant Science, 66, 20-26.

Gholami, A., Shahsavani, S. \& Nezarat, S. 2009. The effect of plant growth promoting rhizobacteria (PGPR) on germination, seedling growth and yield of maize. International Journal of Biological Sciences, $1,35-40$

Iqbal, J. \& Rafique, N. 1987. Toxic effects of bacl2 on germination, early seedling growth, soluble-proteins and acid-phosphatases in Zea mays-L. Pakistan Journal of Botany, 19, 1-8.

Ke, P. J., Miki, T. \& Ding, T. S. 2015. The soil microbial community predicts the importance of plant traits in plant-soil feedback. New Phytologist, 206, 329-341.

Kloepper, J. W., Ryu, C.-M. \& Zhang, S. 2004. Induced systemic resistance and promotion of plant growth by Bacillus species. Phytopathology, 94, 1259-1266.

Kuan, K. B., Othman, R., Rahim, K. A. \& Shamsuddin, Z. H. 2016. Plant Growth-Promoting Rhizobacteria Inoculation to Enhance Vegetative Growth, Nitrogen Fixation and Nitrogen Remobilisation of Maize under Greenhouse Conditions. Public Library of Science one,11, 1-10 
Li, Y., Xu, S., Gao, J., Pan, S. \& Wang, G. 2016. Bacillus subtilis-regulation of stomatal movement and instantaneous water use efficiency in Vicia faba. Plant Growth Regulation, 78, 43-55.

Liu, Y., Chen, L., Zhang, N., Li, Z., Zhang, G., Xu, Y., Shen, Q. \& Zhang, R. 2016. Plant-Microbe Communication Enhances Auxin Biosynthesis by a Root-Associated Bacterium, Bacillus amyloliquefaciens SQR9. Molecular Plant-Microbe Interactions, 29, 324-330.

Martinez-Viveros, O., Jorquera, M., Crowley, D., Gajardo, G. \& Mora, M. 2010. Mechanisms and practical considerations involved in plant growth promotion by rhizobacteria. Journal of Soil Science and Plant Nutrition, 10, 293-319.

Ndeddy Aka, R. J. \& Babalola, O. O. 2016. Effect of bacterial inoculation of strains of Pseudomonas aeruginosa, Alcaligenes feacalis and Bacillus subtilis on germination, growth and heavy metal (cd, cr, and ni) uptake of Brassica juncea. International Journal of Phytoremediation, 18, 200-209.

Nkebiwe, P. M., Weinmann, M. \& Müller, T. 2016. Improving fertilizer-depot exploitation and maize growth by inoculation with plant growth-promoting bacteria: from lab to field. Chemical and Biological Technologies in Agriculture, 3, 1-16.

Ortíz-Castro, R., Contreras-Cornejo, H. A., Macías-Rodríguez, L. \& López-Bucio, J. 2009. The role of microbial signals in plant growth and development. Plant Signaling \& Behavior, 4, 701-712.

Pieterse, C. M., De Jonge, R. \& Berendsen, R. L. 2016. The soil-borne supremacy. Trends in Plant Science, 21, 171-173.

Saha, M., Maurya, B. R., Meena, V. S., Bahadur, I. \& Kumar, A. 2016. Identification and characterization of potassium solubilizing bacteria (KSB) from Indo-Gangetic Plains of India. Biocatalysis and Agricultural Biotechnology, 7, 202-209

Shalaby, S. \& Horwitz, B. A. 2015. Plant phenolic compounds and oxidative stress: integrated signals in fungal-plant interactions. Current Genetics, 61, 347-357.

Shanmugam, V., Kanoujia, N., Singh, M., Singh, S. \& Prasad, R. 2011. Biocontrol of vascular wilt and corm rot of gladiolus caused by Fusarium oxysporum f. sp. gladioli using plant growth promoting rhizobacterial mixture. Crop Protection, 30, 807-813.

Thilagar, G., Bagyaraj, D. \& Rao, M. 2016. Selected microbial consortia developed for chilly reduces application of chemical fertilizers by $50 \%$ under field conditions. Scientia Horticulturae, 198, $27-$ 35.

Tian, J. \& Liao, H. 2015. The role of intracellular and secreted purple acid phosphatases in plant phosphorus scavenging and recycling. Annu Annual Review of Plant Biology, 48, 265-287. 
Vafadar, F., Amooaghaie, R. \& Otroshy, M. 2014. Effects of plant-growth-promoting rhizobacteria and arbuscular mycorrhizal fungus on plant growth, stevioside, NPK, and chlorophyll content of Stevia rebaudiana. Journal of Plant Interactions, 9, 128-136.

Vries, F. T. \& Bardgett, R. D. 2016. Plant community controls on short-term ecosystem nitrogen retention. New Phytologist, 210(3), 861-74.

Ye, J., Wang, S., Deng, X., Yin, L., Xiong, B. \& Wang, X. 2016. Melatonin increased maize (Zea mays L.) seedling drought tolerance by alleviating drought-induced photosynthetic inhibition and oxidative damage. Acta Physiologiae Plantarum, 38, 1-13. 